\title{
Probing the timing network: a continuous theta burst stimulation study of temporal categorization
}

\author{
Juan Carlos Méndez ${ }^{1,2,4}$, Lorenzo Rocchi ${ }^{2,3}$, Marjan Jahanshahi ${ }^{2}$, John \\ Rothwell ${ }^{2}$, Hugo Merchant ${ }^{1}$
}

1. Departamento de Neurobiología Conductual y Cognitiva, Instituto de Neurobiología, Universidad Nacional Autónoma de México, Campus Juriquilla, Querétaro, México.

2. Sobell Department of Motor Neuroscience and Movement Disorders, UCL Institute of Neurology, London, United Kingdom.

3. Dipartimento di Neurologia e Psichiatria, Università di Roma "Sapienza", Rome, Italy

4. Present address: Department of Physiology, Anatomy and Genetics, University of Oxford, Oxford, United Kingdom

Correspondence should be addressed to:

Dr. Hugo Merchant, E-mail: hugomerchant@unam.mx

or to

Dr. Juan Carlos Méndez

E-mail: juan.mendeznunez@dpag.ox.ac.uk 


\begin{abstract}
Time perception in the milliseconds and seconds ranges is thought to be processed by different neural mechanisms. However, whether there is a sharp boundary between these ranges and whether they are implemented in the same, overlapped or separate brain areas is still not certain. To probe the role of the right dorsolateral prefrontal cortex (dlPFC), the right supplementary motor area (SMA), and the cerebellum on time perception, we temporarily altered their activity on healthy volunteers on separate sessions using transcranial magnetic stimulation with the continuous Theta Burst Stimulation (cTBS) protocol. A control session was reserved for the stimulation of the primary somatosensory cortex (S1). Before and after stimulation, participants were tested on temporal categorization tasks using intervals in the hundreds and thousands of milliseconds ranges, as well as on a pitch categorization task which was used as a further control. We then looked for changes in the Relative Threshold and the Constant Error, which, respectively, reflect participants' sensitivity to interval duration and their accuracy at setting an interval that acts as a boundary between categories. We found that after cTBS in all of the studied regions, the Relative Threshold, but not the Constant Error, was affected and only when hundreds of milliseconds intervals were being categorized. Categorization of thousands of milliseconds intervals and of pitch was not affected. These results suggest that the fronto-cerebellar circuit is particularly involved in the estimation of intervals in the hundreds of milliseconds range.
\end{abstract}




\section{Introduction}

Temporal information is present in every perceived stimulus. Yet, current evidence suggests that different brain mechanisms have evolved in humans and other species to quantify durations in scales ranging from microseconds to circadian rhythms (Buonomano and Karmarkar, 2002; Mauk and Buonomano, 2004; Merchant and de Lafuente, 2014). For example, it is well accepted that the microsecond difference in the time of arrival of a sound wave to the two ears is used by brainstem nuclei to compute the localization of the sound source (Brand et al., 2002; Grothe, 2003). In contrast, daily variations in light are used to calibrate circadian rhythms through the hypothalamic suprachiasmatic nuclei (Dibner et al., 2010). Between these two scales, the quantification of intervals in the milliseconds and seconds ranges is less clear. The dorsolateral prefrontal cortex (dlPFC), the supplementary motor area (SMA), the basal ganglia, and the thalamus are emerging as key structures of a core timing circuit (Merchant et al., 2013a, 2015). However, given that a wide variety of behaviors require timing, ranging from the estimation of the duration of stimuli to the production of rhythmic movements, other brain areas, such as the sensory cortices and the cerebellum, might be recruited depending on the particular task requirements (Merchant et al., 2008; Stauffer et al., 2012). Furthermore, while some of these areas appear to participate in the estimation of both, millisecond and seconds-long intervals, others may be more specific (Lewis and Miall, 2003a, 2003b; Hayashi et al., 2014).

Most of the studies that have addressed these problems have relied on correlative approaches, whether observing whole brain activity through neuroimaging (Rao et al., 2001; Lewis and Miall, 2003a, 2003b; Jahanshahi et al., 2006) or scalp electrodes (Jongsma et al., 2003; Honing et al., 2012), by measuring the responses of single cells (Leon and Shadlen, 2003, Merchant et al., 2013b, 2015; Crowe et al., 2014), local field potentials (Bartolo et al., 2014; Bartolo and Merchant, 2015), or through psychophysical techniques (Merchant et al., 2008; Cicchini et al., 2012; Rammsayer and Troche, 2014). In contrast, Transcranial Magnetic

Stimulation (TMS) is a powerful tool that can transiently alter cortical activity, allowing researchers to causally address the role of different sites on motor, sensory and cognitive abilities in healthy human subjects (Walsh and Cowey, 2000; Ridding and Rothwell, 2007). 
Consequently, many studies have begun to use this tool to test whether some of the aforementioned regions are relevant for time production (Theóret et al., 2001; Koch et al., 2003, 2007; Jones et al., 2004; Doumas et al., 2005; Del Olmo et al., 2007; Oliveri et al., 2009; Giovannelli et al., 2014a) or perception (Lee et al., 2007; Giovannelli et al., 2014b).

Among the different stimulation protocols for cortical manipulation with TMS, the theta burst stimulation (TBS) protocol has several advantages: the stimulation period is very short (less than one minute) and its effect lasts up to an hour (Huang et al. 2005), more than enough to test participants on timing tasks. The continuous version of TBS (cTBS) has been shown to inhibit cortical activity (Huang et al., 2005; Stagg et al., 2009; Volman et al., 2011) and alter brain functional connectivity (Volman et al., 2011; Valchev et al., 2015). Indeed, this technique has already been used to probe the role of the premotor cortex (Bijsterbosch et al., 2011a) and the cerebellum (Bijsterbosch et al., 2011b) on the correction of rhythmic movements and of the SMA on single interval reproduction (Dusek et al., 2011). To our knowledge, only one study (Grube et al., 2010) has used cTBS (in the medial cerebellum) to manipulate time perception.

To further probe the role of the right SMA, right dIPFC, and the right cerebellar hemisphere on timing in the hundreds of milliseconds and thousands of milliseconds ranges, we tested human volunteers on an interval categorization task both before and after cTBS on these areas. Categorization is a basic perceptual process whereby stimuli in the environment are assigned to different groups called categories whose members are treated as equal. This is achieved by comparing the stimulus to be categorized with the boundaries between possible categories and/or with their prototypes (Kéri, 2003; Ashby and Maddox, 2005). Therefore, we hypothesized that if the areas studied are essential for timing, their disruption with cTBS would impair either interval perception per se and/or the generation of the interval serving as the boundary between categories. Accordingly, we expected to find differential effects on either the psychophysical Relative Threshold (a measure of categorization variability) and/or on the Constant Error (a measure of the localization of the categorization boundary) depending on the particular role of the tested region. 


\section{Experimental procedures}

\section{Participants}

Fifteen subjects ( 5 female, 10 male; $24.4 \pm 4.85$ (mean \pm SD)) with no history of neurological disorder, alcohol or drug abuse, volunteered for this study. The procedures complied with the Declaration of Helsinki and were explained before commencement of the experiments to every participant, who then signed an informed consent. Subjects remained naïve about the purpose of the study until completion of all sessions.

\section{Tasks}

All the participants performed two blocks of an interval-categorization task and one block of a pitch-categorization task. In the first case, the interval elapsed between two brief (50 ms) tones had to be categorized as short or long (Mendez et al. 2011), whereas in the second case, the pitch of the tones had to be categorized as low or high. The categorized intervals were in the hundreds of milliseconds range (T1: 200, 250, 319, 331, 369, 381, 450, 500) or in the thousands of milliseconds range (T2: 870, 920, 981, 1169, 1231, 1419, 1470, 1520). The tones categorized in the pitch task were: 560, 620, 710, 740, 760, 790, 880 and $940 \mathrm{~Hz}$. In all of these cases the first four intervals (or tones) were considered short (or low) and the remaining four were considered long (or high). In the interval-categorization tasks the pitch was the same for all trials $(1,000 \mathrm{~Hz})$, whereas in the pitch categorization task it was the interval which was kept constant (1 s). The tones were delivered through the laptop's speakers and the participants were allowed to adjust the volume to a comfortable level. Participants responded by pressing on the keyboard the number 1 if they thought the interval was short or the tone was low, and key 2 otherwise. It took approximately 18 minutes to complete all three tasks, which were performed before stimulation and repeated 5 minutes after it to allow for the full effect of cTBS to develop (Huang et al., 2005). The order in which the tasks were performed both before and after stimulation was randomized in each session.

A training phase, consisting of 16 trials, was done in the beginning of each task. In this phase only the shortest and the longest intervals (or the lowest and the highest pitch tones) were presented alternately. The phrase "short (or low), press 1", "long (or high), press 2" appeared 
as soon as the two tones had been presented. Feedback was provided with the words "correct" or "incorrect". The testing phase, consisting of 8 trials with each of the 8 intervals presented randomly for a total of 64 trials, was performed immediately after the training phase. In this phase neither instructions nor feedback were given. Participants were instructed to press the key as soon as they had reached a decision without overthinking.

\section{Experimental sessions}

Every participant completed four sessions on different days, one for each area tested (right SMA, right dIPFC, right cerebellar hemisphere, right S1), with the order counterbalanced across participants. The sessions' dates and times were decided by the participants but at least two days elapsed between each. Usually it took between one to two weeks to complete all four sessions.

\section{Transcranial Magnetic Stimulation}

We used the cTBS protocol to interfere with the activity of the right dIPFC, SMA, and the right cerebellar hemisphere in separate sessions. Magnetic pulses were delivered using a Magstim Rapid apparatus (Magstim Co., Whitland, Wales, UK) with a hand-held coil. For the SMA, a double cone coil with an inner diameter of $90 \mathrm{~mm}$ was used, whereas for the rest of the structures we used a standard figure-of-eight coil with $70 \mathrm{~mm}$ of inner diameter. A detailed description of the cTBS protocol can be found elsewhere (see Huang et al., 2005). Briefly, trains of three pulses delivered at $50 \mathrm{~Hz}$ and separated from each other by $200 \mathrm{~ms}$ were delivered for $40 \mathrm{~s}$, for a total of 600 pulses. The intensity was adjusted individually in all sessions to be $80 \%$ of the active motor threshold (AMT) of the contralateral Tibialis Anterior (TA) muscle in the case of SMA stimulation or of the contralateral First Dorsal Interosseous (FDI) in the case of the other structures. The AMT is defined as the minimum single TMS pulse intensity required to evoke a motor evoked potential larger than $200 \mu \mathrm{V}$ in 5 out of 10 trials while the subject maintains a voluntary contraction of the target muscle of $20 \%$ of the maximal with the help of visual feedback from the surface electromyogram (Huang et al., 2005). Since stimulation in S1 was done as a control, stimulation intensity was $60 \%$ of AMT for this region only. 
Localization of the stimulation site for all the structures was done using well-established craniometric techniques. The dlPFC was localized $5 \mathrm{~cm}$ anterior to the FDI hotspot (George et al., 1995; Zheng, 2000; Epstein et al., 2002; Jones et al., 2004), whereas SMA was considered to be $4 \mathrm{~cm}$ anterior to the hotspot of TA (Steyvers et al., 2003; Hamada et al., 2009), both in the right hemisphere. The right cerebellar hemisphere was found measuring 1 $\mathrm{cm}$ below and $3 \mathrm{~cm}$ to the right of the inion (Théoret et al., 2001; Koch et al., 2007) and was stimulated with the handle of the coil pointing upwards. Finally, S1 was found by measuring $2 \mathrm{~cm}$ posterior to the FDI motor hotspot (Enomoto et al., 2001; Ishikawa et al., 2007; Katayama et al., 2010; Rai et al., 2012).

\section{Data Analysis}

Subroutines written in Matlab (Matworks v. 7.6.0.324) and the SPSS statistical package were used for statistical analyses. The relative threshold was calculated for each subject and for each block of trials from the subject's psychometric curve, where the probability of longinterval categorization was plotted as a function of the stimulus magnitude. A logistic function was fitted to these data, and the relative threshold was computed as half the subtraction of the interval or the distance at $0.75 \mathrm{p}$ and at $0.25 \mathrm{p}$. This represents a measure of the amount of change that a stimulus must have in the studied dimension to be detected by the subject (Gescheider, 1997). In addition, the point of subjective equality (PSE) was calculated as the interval or distance at $0.5 \mathrm{p}$ in the psychometric curves and was then used to calculate the constant error (CE) as:

$$
\mathrm{CE}=\mathrm{PSE}-\text { implicit limit, }
$$

where the implicit limit was the interval or tone that lied in the middle between the shortest (or highest) and the longest (or lowest) categorized values and which was not actually presented to the participants but was expected to be acquired implicitly during the training phase. Thus, the constant error is a measure of how accurate the participant is at setting the limit between categories (Gescheider, 1997). The relative threshold and the constant error were then used as the main variables on two-way repeated measures ANOVAs with stimulation Site (dIPFC, SMA, cerebellum, S1) and cTBS (pre, post -cTBS) as factors 
separately for each task. To confirm that there was no effect derived from learning across sessions, we also performed a one-way repeated measures ANOVA with the sessions as factor on the pre-cTBS performance, defined as the percentage of correct trials. The reported P-values in all the repeated measures ANOVAs correspond to the Greenhouse-Geisser test, which corrects for possible deviations in sphericity.

\section{Results}

All but one participant performed the interval and pitch categorization tasks with more than 70 percent correct trials before cTBS. Thus the analyses reported here were done without this participant's data. As expected, the ANOVAs on subject's performance showed that it did not change across sessions for any of the tasks (Table 1; Figure 1), confirming no effects of session order or practice.

None of the two-way repeated measures ANOVAs with Site and cTBS as factors showed any significant two-way interactions (Table 2). However, the Relative Threshold on the T1 task was significantly affected by the application of cTBS $(F(1,13)=9.309, p=0.009)$. This can be seen in Figure 2, in which the slope of the psychometric curve for the T1 task clearly decreases after the application of cTBS, something that does not happen for the other two tasks. There were no significant effects depending on the stimulation Site (Table 2).

\section{Discussion}

Using a wide variety of neuroscientific tools, the cerebellum (Schubotz et al. 2000; Baer et al. 2005; Pecenka et al. 2013), the dlPFC (Onoe et al., 2001; Genovesio et al. 2006, 2015a, 2015b) and the SMA (Crowe et al., 2014; Merchant et al., 2014) have been shown to be involved in time perception and production in the hundreds and thousands of milliseconds ranges. However, it is not known if they mainly process intervals within a particular range and which specific roles they play. Therefore, we reasoned that interfering with these regions' activity with continuous Theta Burst Stimulation (cTBS) could introduce noise to the timing network and affect participants' ability to categorise intervals in either the milliseconds, the 
seconds, or both ranges. We tested human participants on a time categorization task before and after the application of cTBS and looked for changes in their psychophysical Relative Threshold and Constant Error (CE). The former variable reflects participants' sensitivity to the passage of time, whereas the latter estimates their accuracy for setting the boundary between categories (Gescheider, 1997). As controls, we also stimulated S1 and tested subjects on a pitch categorization task. We found that after cTBS the categorization of intervals in the milliseconds range was affected, unlike that of intervals in the seconds range and of auditory tones which was not affected at all. Furthermore, this change was observed only on the Relative Threshold and not on the CE. Surprisingly, there were no significant differences between the tested regions.

These results have several implications. First of all, the differential effect of cTBS on the categorization of milliseconds and seconds means that, either the structures affected by cTBS only participate in the processing of the former duration range, or that, even if they also have a role in second-range estimation, the network responsible for this is more resilient to the disruption of these nodes than the millisecond-range one. In any case, our results support the notion of separate neural mechanisms and/or circuits for the processing of intervals in the hundreds and thousands of milliseconds ranges (Mauk and Buonomano, 2004; Buhusi and Meck, 2005). Furthermore, they suggest that indeed there exists a thin boundary between the two mechanisms, located somewhere between 800 to 1,500 milliseconds, as already implied by other findings (Gibbon et al., 1997; Rammsayer, 1999; Koch et al., 2009; Gooch et al., 2011). The specific durations we tested was necessarily arbitrary and a more dedicated search for this millisecond-second boundary, using a greater number of intervals, will help to sharpen its exact location and differentiate between the mechanisms associated with each range.

Now, the fact that the Relative Threshold was affected but not the Constant Error further suggests that, even for timing within the millisecond range, there were also two separable processes taking place, namely, an estimation of the duration of the interval presented on each trial, and also, the generation and storage of a mental 'implicit' duration which acted as the limit between interval categories. Whereas the former process was impaired by cTBS, the 
latter was not. Once more, there are two plausible explanations for this finding: either the structures probed do not participate in the storage of durations that will then be used as a reference for categorization, or this process can still take place after the acute disruption of one of its nodes. Presumably, working memory would be involved in the maintenance of duration information, which would suggest that the dlPFC and the posterior parietal cortex would be recruited (Koch et al., 2009). The fact that cTBS to the dlPFC did not affect the CE in any of the categorization tasks supports the interpretation of a strong network, capable of storing information (Mackey et al., 2016) and maintaining the capacity to categorize stimuli (Minamimoto et al., 2010) despite the loss of the dlPFC.

Finally, it was unexpected to find that the effect of cTBS on the Relative Threshold of the millisecond categorization was not different depending on the structure stimulated, including our control structure, S1. This should be interpreted with caution, but we think that this adds up to the evidence showing that timing in the millisecond range happens through a distributed network with no sole node playing a crucial role (Merchant et al., 2013). While timing was affected in our participants, they still were able to maintain a decent performance, a finding echoing reports of patients with injuries to different brain structures still retaining the capacity to display timed behaviors (Aparicio et al. 2005; Coslett et al. 2010; Gooch et al. 2011). This could also imply that several brain structures possess the sufficient neural architecture to support temporal estimation, endowing the timing system with great resilience (Wiener et al. 2011; Merchant et al. 2013).

TMS has been used previously to test the role of different structures on timing, albeit resorting to tasks, durations, and TMS protocols that vary widely. We are, to the best of our knowledge, the first to test interval categorization using cTBS. We are also the first to use TMS to assess the role of the dIPFC on an interval perception task, although its role on interval reproduction tasks has been addressed before (Jones et al. 2004; Koch et al. 2007). Paradoxically, these latter studies failed to find an effect of TMS on the reproduction of intervals in the hundreds of milliseconds range but found an impairment for the reproduction of durations of 2 seconds or longer (also see Koch et al. 2003). Together with our results, this could be interpreted as a perception-of- milliseconds and production-of-seconds role for the 
dlPFC. Nevertheless, on top of considering task differences, it is essential to take into account the stimulation protocols used; the studies above used repetitive TMS (rTMS) instead of cTBS. A recent report (Benali et al. 2011) showed at the neurophysiological and molecular level that these protocols have different effects on cortical activity, which might also account for the apparently discrepant results.

Similar considerations should be taken with studies showing that off-line (Del Olmo et al. 2007; Giovannelli et al. 2014a) and on-line (Jones et al. 2004) rTMS to the SMA does not seem to affect the reproduction of intervals in the milliseconds range. In this case, attention should also be paid to the type of coil used for stimulation: in the referred studies a standard figure of eight coil was used, whereas we used a double-cone coil which is considered to deliver pulses that penetrate further into the cortical tissue (Deng et al. 2014) albeit with a wider spread (Deng et al. 2013). Additionally, whereas Jones et al. (2004) and the present study used leg muscles as a reference to calibrate the intensity for stimulation, Del Olmo et al. (2007) and Giovannelli et al. (2014a) used the FDI. Whether our choice of coil and intensity calibration proved more effective is another interesting topic to investigate. On the other hand, the only time perception study probing the SMA (Giovannelli et al., 2014b) used an interval discrimination task with durations between 800 and 1,200 seconds, similar to the ones in our T2 categorization block. As in our case, they found no effect of SMA disruption, giving further support to the role of this area for the perception of shorter intervals.

The role of the cerebellum on millisecond timing seems less controversial. In accordance with our findings, on-line and off-line rTMS on this region provokes changes on temporal categorization (Lee et al. 2007), reproduction (Koch et al. 2007) or finger tapping (Del Olmo et al. 2007) in the hundreds of milliseconds range but not in the seconds range. Interestingly, one study found no effect of right cerebellar hemisphere cTBS stimulation on a finger tapping task in the sub-seconds range (Bijsterbosch et al. 2011b), although an increase in this structure's activity observed with fMRI is acknowledged in this same report. Once more, the intensity calibration method used in this study differed from ours, as they used the thumb movement visualization method whereas we used the AMT with the aid of the electromyogram (see Methods; Huang et al. 2005). 


\section{Conclusions}

Overall, our results strengthen the notion of distinct mechanisms for the perception of intervals in the millisecond and second range intervals, with a boundary between them near 1 second. Whereas more and more evidence points to the existence of a timing network comprised of several distinct brain regions (Merchant et al., 2013), some of which likely work as a substrate for the processing of both milliseconds and seconds, the dlPFC, the SMA and the cerebellum seem particularly relevant for the estimation of intervals in the former range. Our results also suggest that different cognitive abilities that depend on timing recruit different circuits, with at least one circuit dedicated to the active estimation of intervals, and another, perhaps more general, to the formation and storage of durations for a later use. On another hand, the differences between our findings and previous reports highlight that the choice of TMS protocol and tasks can have a huge impact on the outcome of these experiments (Huang et al. 2011; Hamada et al. 2013). Thus, our interpretations need to be complemented by more experimental results using TMS together with other techniques. Importantly, the tasks need to be comparable and the durations tested must span a wider range before reaching more sound conclusions about the roles of different brain regions in timing in the sub and supra-seconds range. 


\section{Acknowledgements}

We thank Thalia Harmony for sharing the TMS facilities at the INB, and Raul Paulín and Luis Prado for their technical assistance. Supported by CONACYT: 236836 and 196, PAPIIT: IN201214-25 grants to Hugo Merchant. Juan Carlos Méndez is currently a

Postdoctoral Research Scientist at the Department of Physiology, Anatomy and Genetics, University of Oxford.

\section{Conflict of interest}

The authors have no conflict of interests to declare. 


\section{Figure Legends.}

Figure 1. Mean $( \pm \mathrm{SEM})$ performance of all participants across sessions for the three tasks.

Figure 2. Mean $( \pm$ SEM) psychometric curves of all participants before (black solid line) and after cTBS (grey solid line) to the right SMA, dlPFC, S1 and the right cerebellar hemisphere. The change in the Relative Threshold after cTBS in the hundreds of milliseconds categorization task (T1, top) is evident as a decrease in the slope of the grey curve, which is not seen in the thousands of milliseconds (T2, middle) or the Pitch (bottom) tasks. Whiskers $= \pm 1$ SEM. 


\section{References}

Aparicio P, Diedrichsen J, Ivry RB (2005) Effects of focal basal ganglia lesions on timing and force control. Brain Cogn 58: 62-74.

Ashby FG, Maddox WT (2005) Human category learning. Annu Rev Psychol 56:149-78.

Baer LH, Park MT, Bailey JA, Chakravarty MM, Li KZ, Penhune VB (2015) Regional cerebellar volumes are related to early musical training and finger tapping performance. Neuroimage 109: 130-9.

Bartolo R, Prado L, Merchant H (2014) Information processing in the primate basal ganglia during sensory guided and internally driven rhythmic tapping. J Neurosci 34: 3910-23.

Bartolo R, Merchant H (2015) Beta oscillations are linked to the initiation of sensory-cued movement sequences and the internal guidance of regular tapping in the monkey. J Neurosci 35: 4635-40.

Benali A, Trippe J, Weiler E, Mix A, Petrasch-Parwez E, Girzalsky W, Eysel UT, Erdmann R, Funke K (2011) Theta-burst transcranial magnetic stimulation alters cortical inhibition. J Neurosci 31: 1193-203.

Bijsterbosch JD, Lee KH, Dyson-Sutton W, Barker AT, Woodruff PW (2011a) Continuous theta burst stimulation over the left pre-motor cortex affects sensorimotor timing accuracy and supraliminal error correction. Brain Res 1410: 101-11.

Bijsterbosch JD, Lee KH, Hunter MD, Tsoi DT, Lankappa S, Wilkinson ID, Barker AT, Woodruff PW (2011b) The role of the cerebellum in sub- and supraliminal error correction during sensorimotor synchronization: evidence from fMRI and TMS. J Cogn Neurosci 23: $1100-12$. 
Brand A, Behrend O, Marquardt T, McAlpine D, Grothe B (2002) Precise inhibition is essential for microsecond interaural time difference coding. Nature 417: 543-7.

Buhusi CV, Meck WH (2005) What makes us tick? Functional and neural mechanisms of interval timing. Nat Rev Neurosci. 6: 755-65.

Buonomano DV, Karmarkar UR (2002) How do we tell time? Neuroscientist 8: 42-51.

Coslett HB, Wiener M, Chatterjee A (2010) Dissociable neural systems for timing: evidence from subjects with basal ganglia lesions. PLoS One 5(4):e10324.

Cicchini GM, Arrighi R, Cecchetti L, Giusti M, Burr DC (2012) Optimal encoding of interval timing in expert percussionists. J Neurosci 32: 1056-60.

Crowe DA, Zarco W, Bartolo R, Merchant H (2014) Dynamic representation of the temporal and sequential structure of rhythmic movements in the primate medial premotor cortex. $\mathrm{J}$ Neurosci 34: 11972-11983.

Del Olmo MF, Cheeran B, Koch G, Rothwell JC (2007) Role of the cerebellum in externally paced rhythmic finger movements. J Neurophysiol 98: 145-52.

Deng ZD, Lisanby SH, Peterchev AV (2014) Coil design considerations for deep transcranial magnetic stimulation. Clin Neurophysiol 125: 1202-12.

Deng ZD, Lisanby SH, Peterchev AV (2013) Electric field depth-focality tradeoff in transcranial magnetic stimulation: simulation comparison of 50 coil designs. Brain Stimul 6: $1-13$.

Dibner C, Schibler U, Albrecht U (2010) The mammalian circadian timing system: organization and coordination of central and peripheral clocks. Annu Rev Physiol 72: 51749. 
Doumas M, Praamstra P, Wing AM (2005) Low frequency rTMS effects on sensorimotor synchronization. Exp Brain Res 167: 238-45.

Dusek P, Jech R, Havrankova P, Vymazal J, Wackermann J (2011) Theta-burst transcranial magnetic stimulation over the supplementary motor area decreases variability of temporal estimates. Neuro Endocrinol Lett 32: 481-6.

Epstein CM, Sekino M, Yamaguchi K, Kamiya S, Ueno S (2002) Asymmetries of prefrontal cortex in human episodic memory: effects of transcranial magnetic stimulation on learning abstract patterns. Neurosci Lett 320: 5-8.

Genovesio A, Cirillo R, Tsujimoto S, Mohammad Abdellatif S, Wise SP (2015a) Automatic comparison of stimulus durations in the primate prefrontal cortex: the neural basis of acrosstask interference. J Neurophysiol 114: 48-56.

Genovesio A, Seitz LK, Tsujimoto S, Wise SP (2015b) Context-Dependent Duration Signals in the Primate Prefrontal Cortex. Cereb Cortex 26: 3345-56.

Genovesio A, Tsujimoto S, Wise SP (2006) Neuronal activity related to elapsed time in prefrontal cortex. J Neurophysiol 95: 3281-5.

George MS, Wassermann EM, Williams WA, Callahan A, Ketter TA, Basser P, Hallett M, Post RM (1995) Daily repetitive transcranial magnetic stimulation (rTMS) improves mood in depression. Neuroreport 6: 1853-6.

Gescheider GA (1997) Psychophysics: The Fundamentals. Third edition. Lawrence Erlbaum Associates. New Jersey.

Gibbon J, Malapani C, Dale CL, Gallistel C (1997) Toward a neurobiology of temporal cognition: advances and challenges. Curr Opin Neurobiol 7: 170-84. 
Giovannelli F, Innocenti I, Rossi S, Borgheresi A, Ragazzoni A, Zaccara G, Viggiano MP, Cincotta M (2014a) Role of the dorsal premotor cortex in rhythmic auditory-motor entrainment: a perturbational approach by rTMS. Cereb Cortex 24: 1009-16.

Giovannelli F, Ragazzoni A, Battista D, Tarantino V, Del Sordo E, Marzi T, Zaccara G, Avanzini G, Viggiano MP, Cincotta M (2014b) "...The times they aren't a-changin'..." rTMS does not affect basic mechanisms of temporal discrimination: a pilot study with ERPs. Neuroscience 278: 302-12.

Gooch CM, Wiener M, Hamilton AC, Coslett HB (2011) Temporal discrimination of suband suprasecond time intervals: a voxel-based lesion mapping analysis. Front Integr Neurosci $5: 59$.

Grothe B (2003) New roles for synaptic inhibition in sound localization. Nat Rev Neurosci 4: $540-50$.

Grube M, Lee KH, Griffiths TD, Barker AT, Woodruff PW (2010) Transcranial magnetic theta-burst stimulation of the human cerebellum distinguishes absolute, duration-based from relative, beat-based perception of subsecond time intervals. Front Psychol 1: 171.

Hamada M, Hanajima R, Terao Y, Okabe S, Nakatani-Enomoto S, Furubayashi T, Matsumoto H, Shirota Y, Ohminami S, Ugawa Y (2009) Primary motor cortical metaplasticity induced by priming over the supplementary motor area. J Physiol 587: 484562.

Hamada M, Murase N, Hasan A, Balaratnam M, Rothwell JC (2013) The role of interneuron networks in driving human motor cortical plasticity. Cereb Cortex 23: 1593-605.

Hayashi MJ, Kantele M, Walsh V, Carlson S, Kanai R (2014) Dissociable neuroanatomical correlates of subsecond and suprasecond time perception. J Cogn Neurosci 26: 1685-93. 
Honing H, Merchant H, Háden GP, Prado L, Bartolo R (2012) Rhesus monkeys (Macaca mulatta) detect rhythmic groups in music, but not the beat. PLoS One 7(12):e51369.

Huang YZ, Edwards MJ, Rounis E, Bhatia KP, Rothwell JC (2005) Theta burst stimulation of the human motor cortex. Neuron 45: 201-206.

Huang YZ, Rothwell JC, Chen RS, Lu CS, Chuang WL (2011) The theoretical model of theta burst form of repetitive transcranial magnetic stimulation. Clin Neurophysiol 122:1011-8.

Jahanshahi M, Jones CR, Dirnberger G, Frith CD (2006) The substantia nigra pars compacta and temporal processing. J Neurosci 26: 12266-73.

Jones CR, Rosenkranz K, Rothwell JC, Jahanshahi M (2004) The right dorsolateral prefrontal cortex is essential in time reproduction: an investigation with repetitive transcranial magnetic stimulation. Exp Brain Res 158: 366-72.

Jongsma ML, Desain P, Honing H, van Rijn CM (2003) Evoked potentials to test rhythm perception theories. Ann N Y Acad Sci 999: 180-3.

Koch G, Oliveri M, Caltagirone C (2009) Neural networks engaged in milliseconds and seconds time processing: evidence from transcranial magnetic stimulation and patients with cortical or subcortical dysfunction. Philos Trans R Soc Lond B Biol Sci. 364: 1907-18.

Koch G, Oliveri M, Torriero S, Caltagirone C (2003) Underestimation of time perception after repetitive transcranial magnetic stimulation. Neurology 60: 1844-6.

Koch G, Oliveri M, Torriero S, Salerno S, Lo Gerfo E, Caltagirone C (2007) Repetitive TMS of cerebellum interferes with millisecond time processing. Exp Brain Res 179: 291-9. 
Lee KH, Egleston PN, Brown WH, Gregory AN, Barker AT, Woodruff PW (2007) The role of the cerebellum in subsecond time perception: evidence from repetitive transcranial magnetic stimulation. J Cogn Neurosci 19: 147-57.

Leon MI, Shadlen MN (2003) Representation of time by neurons in the posterior parietal cortex of the macaque. Neuron 38: 317-27.

Lewis PA, Miall RC (2003a) Brain activation patterns during measurement of sub- and suprasecond intervals. Neuropsychologia 41: 1583-92.

Lewis PA, Miall RC (2003b) Distinct systems for automatic and cognitively controlled time measurement: evidence from neuroimaging. Curr Opin Neurobiol 13: 250-5.

Mackey WE, Devinsky O, Doyle WK, Meager MR, Curtis CE (2016) Human Dorsolateral Prefrontal Cortex Is Not Necessary for Spatial Working Memory. J Neurosci 36: 2847-56.

Mauk MD, Buonomano DV (2004) The neural basis of temporal processing. Annu Rev Neurosci 27: 307-40.

Mendez JC, Prado L, Mendoza G, Merchant H (2011) Temporal and Spatial Categorization in Human and Non-Human Primates. Front Integr Neurosci 5:50.

Merchant H, Bartolo R, Pérez O, Méndez JC, Mendoza G, Gámez J, Yc K, Prado L (2014) Neurophysiology of timing in the hundreds of milliseconds: multiple layers of neuronal clocks in the medial premotor areas. Adv Exp Med Biol. 829: 143-54.

Merchant H, de Lafuente V (2014) Introduction to the Neurobiology of Interval Timing. Adv Exp Med Biol 829: 1-13. 
Merchant H, Grahn J, Trainer L, Rohrmeier M, Fitch TW (2015) Finding the beat: A neural perspective across humans and non-human primates. Philos Trans R Soc Lond B Biol Sci 370: 186-202.

Merchant H, Harrington DL, Meck WH (2013a) Neural basis of the perception and estimation of time. Annu Rev Neurosci 36: 313-36.

Merchant H, Perez O, Bartolo R, Mendez JC, Mendoza G, Gamez J, Yc K, Prado L (2015) Sensorimotor neural dynamics during isochronous tapping in the medial premotor cortex of the macaque. Eur J Neurosci 41: 586-602.

Merchant H, Pérez O, Zarco W, Gámez J (2013b) Interval tuning in the primate medial premotor cortex as a general timing mechanism. J Neurosci 33: 9082-96.

Merchant H, Zarco W, Pérez O, Prado L, Bartolo R (2011) Measuring time with different neural chronometers during a synchronization-continuation task. Proc Natl Acad Sci U S A 108: 19784-9.

Merchant H, Zarco W, Prado L (2008) Do we have a common mechanism for measuring time in the hundreds of milliseconds range? Evidence from multiple interval timing tasks. J Neurophysiol 99: 939-49.

Minamimoto T, Saunders RC, Richmond BJ (2010) Monkeys quickly learn and generalize visual categories without lateral prefrontal cortex. Neuron 66: 501-7.

Oliveri M, Koch G, Salerno S, Torriero S, Lo Gerfo E, Caltagirone C (2009) Representation of time intervals in the right posterior parietal cortex: implications for a mental time line. Neuroimage 46: 1173-9. 
Onoe H, Komori M, Onoe K, Takechi H, Tsukada H, Watanabe Y (2001) Cortical networks recruited for time perception: a monkey positron emission tomography (PET) study. Neuroimage 13: 37-45.

Pecenka N, Engel A, Keller PE (2013) Neural correlates of auditory temporal predictions during sensorimotor synchronization. Front Hum Neurosci 7:380.

Rammsayer TH (1999) Neuropharmacological evidence for different timing mechanisms in humans. Q J Exp Psychol B 52: 273-86.

Rammsayer TH, Troche SJ (2014) Elucidating the internal structure of psychophysical timing performance in the sub-second and second range by utilizing confirmatory factor analysis. Adv Exp Med Biol 829: 33-47.

Rao SM, Mayer AR, Harrington DL (2001) The evolution of brain activation during temporal processing. Nat Neurosci 4: 317-23.

Ridding MC, Rothwell JC (2007) Is there a future for therapeutic use of transcranial magnetic stimulation? Nat Rev Neurosci 8: 559-67.

Schubotz RI, Friederici AD, von Cramon DY (2000) Time perception and motor timing: a common cortical and subcortical basis revealed by fMRI. Neuroimage 11: 1-12.

Stagg CJ, Wylezinska M, Matthews PM, Johansen-Berg H, Jezzard P, Rothwell JC, Bestmann S (2009) Neurochemical effects of theta burst stimulation as assessed by magnetic resonance spectroscopy. J Neurophysiol 101: 2872-7.

Stauffer CC, Haldemann J, Troche SJ, Rammsayer TH (2012) Auditory and visual temporal sensitivity: evidence for a hierarchical structure of modality-specific and modalityindependent levels of temporal information processing. Psychol Res 76: 20-31. 
Steyvers M, Etoh S, Sauner D, Levin O, Siebner HR, Swinnen SP, Rothwell JC. (2003) Highfrequency transcranial magnetic stimulation of the supplementary motor area reduces bimanual coupling during anti-phase but not in-phase movements. Exp Brain Res 151: 30917.

Théoret H, Haque J, Pascual-Leone A (2001). Increased variability of paced finger tapping accuracy following repetitive magnetic stimulation of the cerebellum in humans. Neurosci Lett 306: 29-32.

Valchev N, Ćurčić-Blake B, Renken RJ, Avenanti A, Keysers C, Gazzola V, Maurits NM (2015) cTBS delivered to the left somatosensory cortex changes its functional connectivity during rest. Neuroimage 114: 386-97.

Volman I, Roelofs K, Koch S, Verhagen L, Toni I (2011) Anterior prefrontal cortex inhibition impairs control over social emotional actions. Curr Biol 21:1766-70.

Walsh V, Cowey A (2000) Transcranial magnetic stimulation and cognitive neuroscience. Nat Rev Neurosci 1: 73-9.

Wiener M, Matell MS, Coslett HB (2011) Multiple mechanisms for temporal processing. Front Integr Neurosci 5:31.

Zheng XM (2000) Regional cerebral blood flow changes in drug-resistant depressed patients following treatment with transcranial magnetic stimulation: a statistical parametric mapping analysis. Psychiatry Res 100: 75-80. 


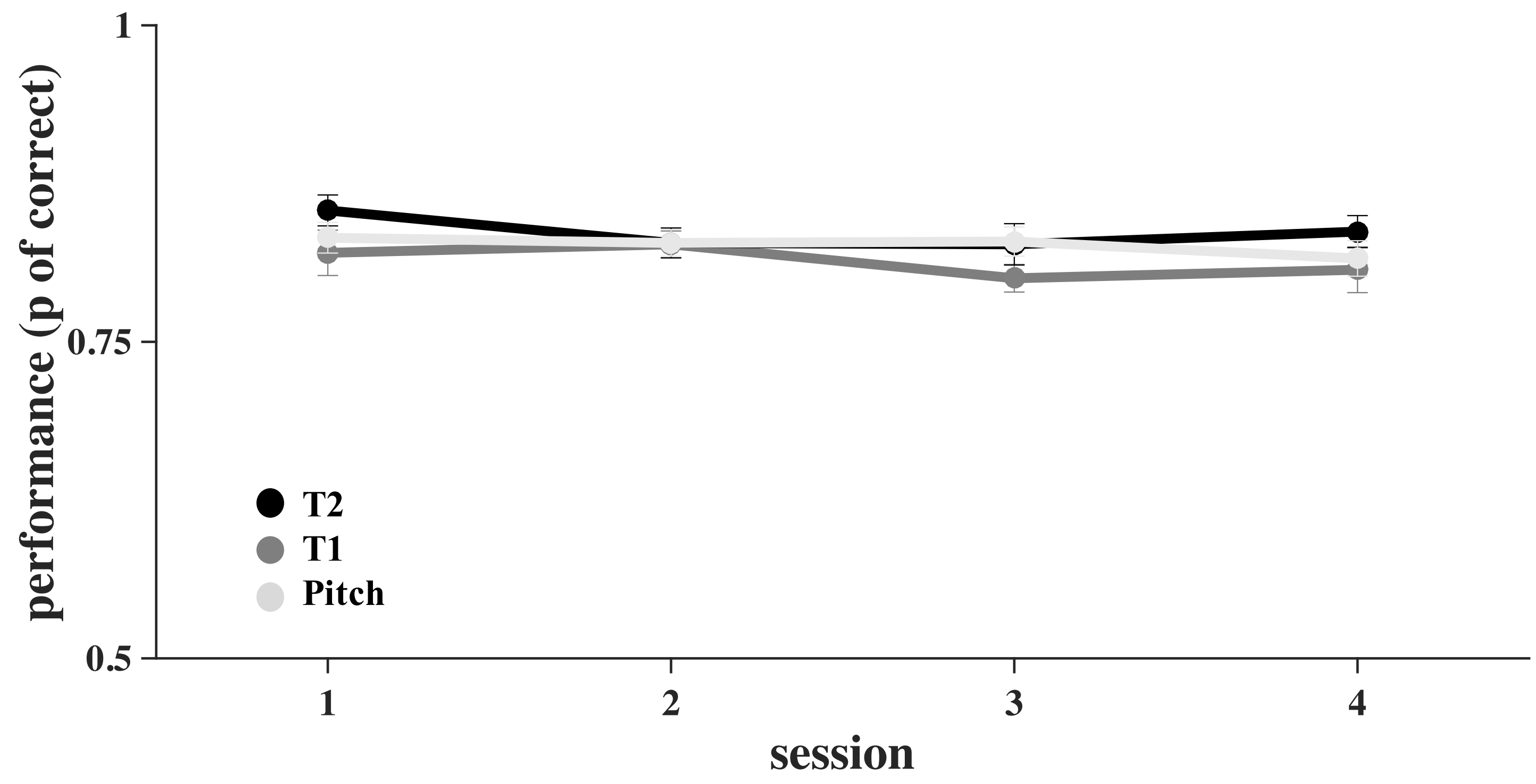

Figure 1 

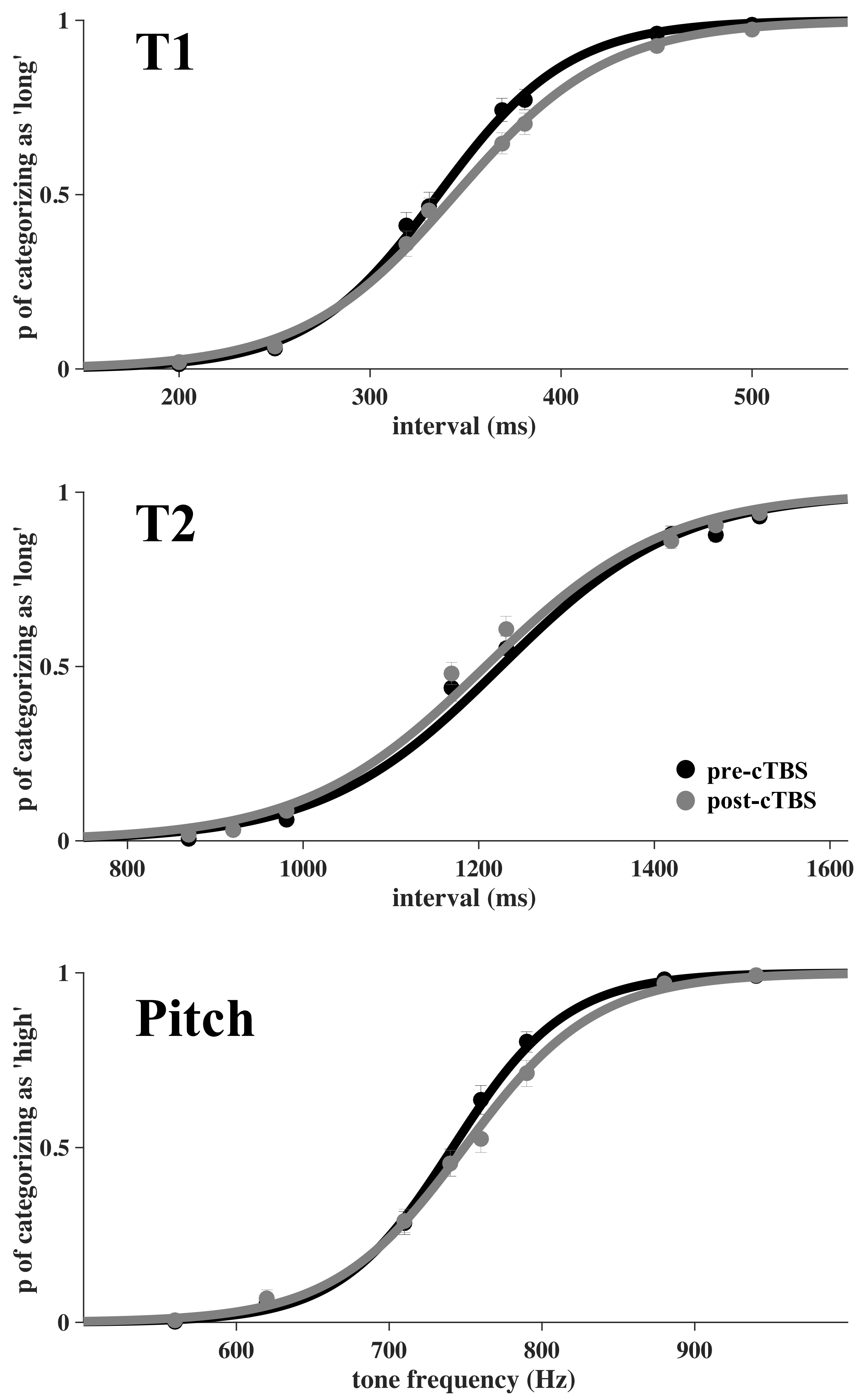